\title{
MEASUREMENT OF ENERGY LOSS OF LIGHT IONS USING SILICON SURFACE BARRIER DETECTOR
}

\author{
Basavaraja Sannakki ${ }^{1}$, Mahalesh Devendrappa ${ }^{2}$ \\ ${ }^{I}$ Professor of Physics, Department of Physics, Gulbarga University, Gulbarga, Karnataka, India \\ ${ }^{2}$ Research Scholar, Department of Physics, Gulbarga University, Gulbarga, Karnataka, India
}

\begin{abstract}
The Energy Loss ( $\triangle E)$ of light ions such as alpha particles [Am-241] of energy $5.48 \mathrm{MeV}$ have been measured in atmospheric gas at different pressures. The energy loss experiment has performed using alpha ray spectrometer. The source of Am-241 that emits alpha particles of He ions of energy $5.48 \mathrm{MeV}$. The passivated ion-implanted planar Silicon Surface Barrier Detector [SSBD] of resolution $20 \mathrm{KeV}$ in vacuum for $5.48 \mathrm{MeV}$ energy. The Alpha Spectrometer constitutes the Multi-Channel Analyzer (MCA-1K) connected with Alpha Ray Spectroscopy Software (ALSS), Power Supply (NIM BIN) and Vacuum Pump. The measured energy loss [DE] in the vacuum at 4.2 mbar was $9.64 \mathrm{KeV}$ and that in without vacuum the energy loss [DE] was $4.005 \mathrm{MeV}$. The range of alpha particles in atmospheric gas at different pressures is obtained through the measurement of transmitted energy.
\end{abstract}

Keywords: Energy Loss, SSBD, Gas Pressure and Transmitted Energy.

\section{INTRODUCTION}

The study of the passage of fast-moving particles through matter has been important, since from the early days of nuclear physics. The light ions, such as alpha particles $\left[{ }^{4} \mathrm{He}\right]$ traversing through a matter, lose their energy primarily thorough the excitation and ionization in the inelastic collision with atomic electrons called as the electronic energy loss (Northcliffe and Schilling 1970, Hubert et al 1990 Ziegler 1999). Microscopically, energy loss due to excitation and ionization is a discrete process. However, macroscopically, it is a good assumption that the moving ions lose their energy, continuously. We considered the average energy loss during the penetration of ions into a given material. The measured energy loss must be determined with the distance $\Delta t$ or the thickness of the thin films. The alpha particles while passing through the target, they lose their energy and the loss of energy depends on the density $(\rho) /$ atomic density $(\mathrm{N})$, thickness of the absorbing target and incident energy as well as the charge state of the projectile ions. The mass density or atomic density gives rise to the energy loss of the target material i.e. $\rho \Delta t$ or $N \Delta t$. Silicon Surface Barrier Detectors (SSBDs) have already been used in the fields of charged particle detection as a target for coherent interaction in a series of experiments by Bellini et al ${ }^{[1,6]}$. Yuan was probably first to use them for detection of charged particles. But, in general the solid state detectors have been used scanty for high energy physics experiments. Because, they cannot provide large detecting area, which is required in common Spectrometers. A need is required now for the thin film particle detector very near to the interaction region with high precision, high rate and high multiplicity capacity, which is difficult to meet with existing detectors. The SSBDs are fabricated with N-type silicon with resistivity in the range $1-8 \mathrm{~K}$ ohm-cm ${ }^{[2]}$. The electrical contacts are made with gold evaporation on the Ptype surface and with aluminum on the N-type back surface provides on ohmic contact detectors is encapsulated with BNC or microdot connector. $\mathrm{Si}$ is one of the extraordinary semiconductors suited for the fabrication of high-quality radiation detectors with a band gap of $1.1 \mathrm{eV}$ at room temperature. The intrinsic concentration of charge carriers is low, thus avoiding excessive noise. The silicon surface barrier (SSB) radiation detectors are widely used in experimental nuclear physics for the spectroscopy of alpha particles, heavy ions, and fission fragments.

\subsection{Surface - Barrier Detectors}

There are three main parameters that define a silicon surfacebarrier detector: resolution, active area, and depletion depth. The ORTEC model numbers reflects these three parameters in its model in the same order. The R-018-450-100 detector which defines its parameters on its model as; The R stands for Ruggedized detector with a resolution of $18 \mathrm{KeV}$ for $241 \mathrm{Am}$ alpha particle, an area of $450 \mathrm{~mm}^{2}$, and a depletion depth of $100 \mu \mathrm{m}$. It operates with $0-200$ bias voltage, which can be varied by ALSS software ${ }^{[3]}$. Figure.1 shows the schematic diagram of experimental set up and circular disc detectors, its active area defines the diameter of its faces at any given distance from the source; a larger area will subtend a larger angle and thus intercept a greater portion to the total number of alpha particles that emanate from the source. The depletion depth is synonymous with the sensitive depth of the detector. For any experiment the depth must be sufficient to completely stop all the charged particles that are to be measured, and its 
ability to do this is dependent upon both the energy and the particle type. Note that a $5.48 \mathrm{MeV}$ alpha particles are completely stopped with about $27 \mu \mathrm{m}$ of silicon. Since, natural alphas are usually less than $8 \mathrm{MeV}$ in energy; a 50- $\mu \mathrm{m}$ detector is adequate to stop all natural alphas. Inadequate, thickness uniformity of totally depleted $\Delta \mathrm{E}$ detectors has undoubtedly been responsible for many disappointing experiments. A $10-\mathrm{MeV}{ }^{4} \mathrm{He}$ particle incident on a $50-\mu \mathrm{m}-$ thick silicon detector will lose approximately $5.9 \mathrm{MeV}$ in traversing the detector. The rate of energy loss $(\mathrm{dE} / \mathrm{dx})$ of the exiting particle, however, will be about $160 \mathrm{KeV} / \mu \mathrm{m}$. This means that a detector thickness variation of $1 \mu \mathrm{m}$ would cause an energy spread of $160 \mathrm{KeV}$.

\subsection{Bohr's Theory of Energy Straggling}

Bohr's (1948) theory is based on the assumption that the incident ions are fully stripped and their energy loss in the target material is very small as compared to the incident ion energy ${ }^{[4,8]}$. According to this theory, the calculations of energy straggling standard deviations, Bohr developed the following expression.

$$
\left(\Omega_{B}\right)^{2}=4 \pi\left(Z_{1}\right)^{2} e^{4} Z_{2} N \text { t }
$$

Where $Z_{1}$ and $Z_{2}$ are the atomic number of incident ion and target material respectively, $e$ is the electronic charge, $N$ is the number of target atoms per unit volume and $t$ is the target thickness.

\subsection{Lindhard and Scharff theory of Energy Straggling}

Lindhard and Scharff (1953) modified the Bohr's theory to make it applicable in the lower energy domain by introducing a velocity dependent factor $\chi$. In accordance with this approach for $\chi \leq 3^{[9]}$

$$
\Omega_{\mathrm{LS}}=0.5 \mathrm{~B} \Omega_{\mathrm{B}}^{2}\left[1.36 \chi^{1 / 2}-0.016 \chi^{3 / 2}\right]
$$

Where, $\chi=v^{2} / v_{0} Z_{2}$ with $v / v_{0}$ is the ratio of ions to Bohr's velocity.

\section{EXPERIMENTAL ARRANGEMNETS}

The energy losses of alpha particles through the atmospheric gas at different pressures have been measured in a vacuum chamber. The experiment was performed using passivated ionimplanted planar SSBD of resolution $20 \mathrm{KeV}$ in vacuum for alpha particles of energy $5.48 \mathrm{MeV}$. The operating voltage of +30 volts has applied to the SSBD detector. The rise time is $<100 \mathrm{~ns}$, fall time is $100 \mu \mathrm{s}$ and output of $+100 \mathrm{mV}$ for 5.15 $\mathrm{MeV}$ for low noise charge sensitive preamplifier. The detector connected with vacuum chamber made with solid brass Nickel plating for ease of decontamination high performance O-ring seal, internal dimensions are $61 \mathrm{~mm}$ wide $\mathrm{x} 74 \mathrm{~mm}$ deep $\mathrm{x}$
$40 \mathrm{~mm}$. Here sample spacing is from $2 \mathrm{~mm}$ minimum to 40 $\mathrm{mm}$ maximum in steps of $4 \mathrm{~mm}$, to control vacuum three positions given PUMP, HOLD and VENT [5].

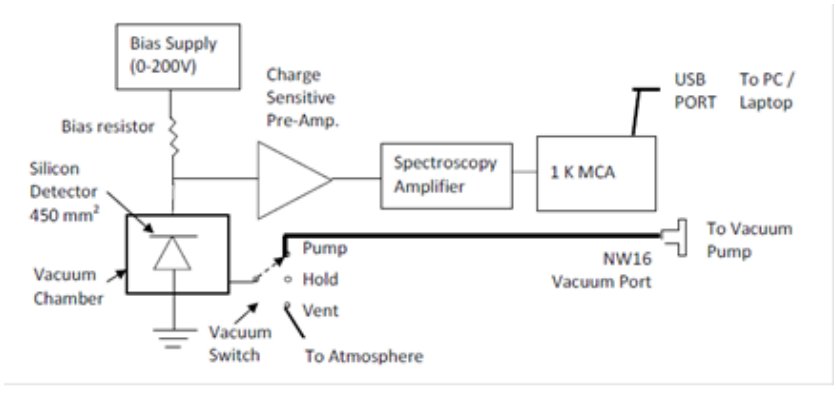

Fig-1: Schematic diagram of experimental set up and circular disc detectors.

The alpha particles with initial energy Eo entering the area of no coating corresponding to signal of energy E1[6]. While, ions penetrating the coated film correspond to signals of energy $\mathrm{E} 2$, then, the energy loss $[\Delta \mathrm{E}]$ of the ions in the thin film can be obtained as follows

$$
\Delta \mathrm{E}=\mathrm{E} 1-\mathrm{E} 2
$$

Hence $\Delta \mathrm{E}$ is the energy loss of the ions through the film or matter.

\subsection{Experimental Procedure}

The energy losses of alpha particles through the atmospheric gas at different pressures have been measured in a vacuum chamber. The alpha source was kept inside the vacuum chamber before creating the vacuum using the positions of HOLD, VENT and PUMP; Then the vacuum was created in the vacuum chamber or scattering chamber, which is connected to a rotary vacuum pump. The alpha source of active area was about $1 \mathrm{~mm}$ diameter and it has been deposited on the geometrical centre of the disk. The Am-241 was used as a source for the alpha particles. The rough vacuum was created by using the rotary pump, initially switched on PC and alpha ray spectrometer [PAS-01] the knob position has kept in vent mode. The alpha source has loaded then the biasing voltage of $30-40 \mathrm{~V}$ has applied for the SSBD detector. The data acquisition time has set to $100 \mathrm{sec}$ then start the acquisition through a window based software ALSS spectroscopy and saved. Now clear the spectra and start fresh acquisition to the next measurements.

The scattering chamber has filled completely with an atmospheric gas/air molecules, where the detector and source are placed then start to create a vacuum in the scattering chamber at pressure of 4.2 mbar and noted down the centre of the peak position in channels and energy has been calibrated using $1 \mathrm{~K}$ multi channel analyzer [MCA] gives $9.64 \mathrm{KeV}$ per channel. The energy loss of alpha particles at different 
pressures in the range of $4.4-9.2$ mbar has been measured. The transmitted energies are also noted down after each pressure to obtain the range of alpha particles in the atmospheric gas.

\section{RESULTS AND DISCUSSION}

The results of experimental measured the energy loss of alpha particles of energy $5.48 \mathrm{MeV}$ in the atmospheric gas at different pressures are discussed. The plot of energy loss of alpha particles as function of pressure of the atmospheric gas is shown in Figure.3. It is observed from Figure. 3 that as the pressure increased the energy loss of alpha particles decreased. At higher pressure the energy loss is less due to increase in pressure indicates that the number of gas molecules present in the scattering chamber decreased and hence the probability of interaction of the alpha particles with gas molecules during traversing is less hence the energy loss is less, consequently the transmitted energy of alpha particles is high. But at lower pressure the energy loss is more due the number of gas molecules present in the scattering chamber is more and hence the probability of interaction of the alpha particles with these gas molecules is more hence the energy loss is less, consequently the transmitted energy of alpha particles is low.

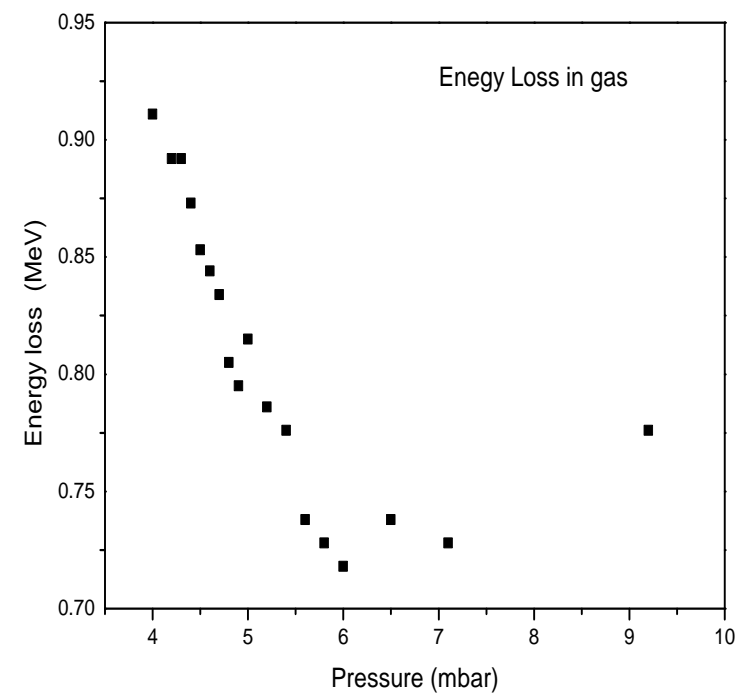

Fig- 2: The plot of energy loss of alpha particles at different pressure of the gas.

Further, the range of the alpha particles in the atmospheric gas at different pressures are has been obtained using the experimental measured values of transmitted energy. The plot of transmitted energy of alpha particles as function of pressure of the atmospheric gas is shown in Figure.4. It is observed from Figure. 4 that as the pressure increased the transmitted energy is also increased and hence the projected range of alpha particles is increased. At higher pressure the energy loss is less due to increase in pressure indicates that the number of gas molecules present in the scattering chamber decreased and hence the probability of interaction of the alpha particles with gas molecules during their traversing is less, hence the particles loses lesser energies, consequently the projected range of transmitted alpha particles is higher. But at lower pressure the energy loss is more due the number density of gas molecules present in the scattering chamber and hence the energy loss is more, consequently the transmitted energy of alpha particles is low hence the range is lower.

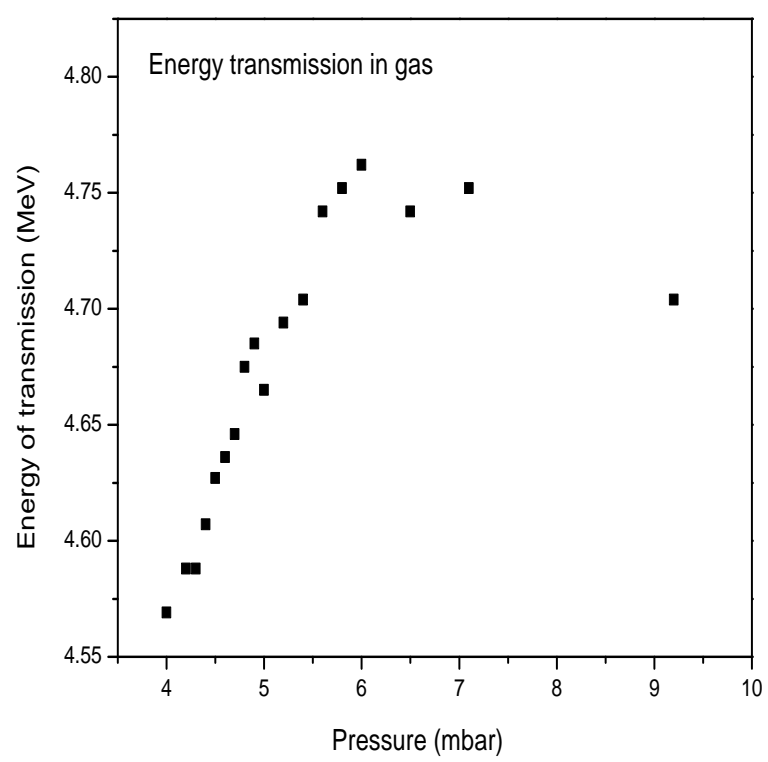

Fig- 3: The plot of transmitted energy of alpha particles at different pressure of the gas.

\section{CONCLUSIONS}

Energy loss of the alpha particles has been measured with and without vacuum at different pressures. The alpha particles of energy $5.48 \mathrm{MeV}$ in the atmospheric gas show that as the pressure increased the energy loss of alpha particles decreased. The range of the alpha particles in the atmospheric gas at different pressures show that as the pressure increased the transmitted energy is also increased and hence the projected range of alpha particles is increased.

\section{ACKNOWLEDGEMENTS}

The authors are acknowledged to the Dept of Physics, Gulbarga University, Gulbarga and the UGC New Delhi for providing a financial assistance to the MRP project. 


\section{REFERENCES}

[1]. G Bellini et al., Nucl. Instr. and Meth. Vol-107 (1973)

[2]. G. F. Knoll, Radiation Detection and Measurement (John Wiley and Sons, New York, p. 354 (1999)

[3]. Alpha Spectroscopy Catalog. (2011) P-4

[4]. P.K. Diwan et al, Radiation Physics and Chemistry, vol81(2012) 1543-1546.

[5]. Alpha spectroscopy user manual (2011) p-1-39.

[6]. E.H.M. Heyne et al., Nucl. Instr. and Meth.vol-178 (1980) p- 331-343

[7]. Bohr, N., 1948. The penetration of atomic particles through matter Kgl. Dan Vid Selsk Mat Fys Medd 18 (8), 1144

[8]. Lindhard, J., Scharff, M., 1953. Energy loss in matter by fast particles of low charge Kgl. Dan Vid Selsk Mat Fys Medd 27 (15), 1-32 